sites have examined the issue. Many cases will prove to be 'false positives'. Other people, even if truly vulnerable to a psychotic disorder, may respond to purely psychosocial interventions or require other forms of drug therapy, for example antidepressants. In others, it may turn out that neuroleptics help to reduce the risk of a first and potentially destructive episode of psychosis. We simply do not yet have sufficient knowledge to be clear about specific treatment-matching at this phase, so further research is urgently required. On the other hand, psychiatrists still need to respond to those in distress and people with substantial levels of disability and risk. For example, in our Personal Assessment and Crisis Evaluation (PACE) clinic which provides assistance for at-risk patients who are actively seeking and accepting help, the rate of deliberate self-harm and disability prior to entry is high (Yung et al, 1996). Better access to interventions for adolescents and young adults with psychosocial disorders is an urgent priority (Rutter \& Smith, 1995); specificity of treatment is essentially a second-order issue.

In first-episode psychosis, the evidence suggests the real problem is excessive caution, with delayed treatment all too common. While it remains to be definitively proven that shortening delays in treatment strongly affects the long-term outcome, the increasing safety and effectiveness of modern treatments support a policy of early intervention once a psychotic illness is clearly present. Obviously, such a proactive stance must respect the rights and wishes of consumers; indeed, in doing so, the chances of a collaborative long-term relationship, the cornerstone of a positive outcome in psychosis, are usually enhanced.

I do not believe that Sullivan either sought to or succeeded in 'nipping this scheme in the bud'. The notion of early intervention is a sound one throughout medicine, provided the maxim primum non nocere is adhered to. Cameron merely argued for this principle to be applied to the most severe disorders that psychiatrists treat. Perhaps the time was not right in the sense that few effective or specific treatments existed then, but the idea was a not a bad one. It is important to separate people from ideas.

Rutter, M. \& Smich, D. f. (eds) (1995) Psychosocial Disorders in Young People: Time Trends and their Couses. Chichester: John Wiley and Sons.

Yung A. R. \& McGorry, P. D. (1997) is pre-psychotic intervention realistic in schizophrenia and related disorders? Australian and New Zealand journal of Psychiotry, 31. $799-805$.

_, , McFarlane, C. A., et al (1996) Monitoring and care of young people at incipient risk of psychosis. Schizophrenio Bulletin, 22, 283-303.

P. McGorry Early Psychosis Prevention and Intervention Centre and Department of Psychiatry, University of Melbourne, Locked Bag 10, Parkville.

Melbourne, Victoria 3052, Australia

\section{Emotional functions and cognitive processes}

Sir: Taylor \& Liberzon (1999) provide a valuable overview of current knowledge of the neurobiology of emotion, and its implications for the study of schizophrenia. One important aspect which was not fully explored is the possible link between emotional function and the capacity for abstract thought, both of which may be abnormal in schizophrenia.

Lesion studies in monkeys indicate the importance of the orbitofrontal cortex in mediating the inhibition of action when this inhibition is required for reward. Damasio (1996) has shown that in man this area is not only essential in inhibiting automatic responses, but also in recognising bodily states associated with reward and punishment. This recognition is necessary to allow the abstraction of rules about which categories of responses are likely to be rewarded. The study quoted used a gambling paradigm. Subjects selected cards at will from two packs; one pack provided rewards of large sums of money, but an overall loss in the course of the experiment, while the second pack produced more modest wins but a consistent gain over the experiment. Subjects with orbitofrontal lesions consistently selected cards from the first pack, whereas those with normal frontal lobe function shifted strategy to select cards from the second pack. Subjects without orbitofrontal lesions were able to 'sense' that one pack was 'better' than the other although they were unable to say why this was.

The suggestion is that in order to be able to shift attention from the immediate consequences of a response (in terms of reward or punishment) to being able to think symbolically about categories of response, systems involved in reward, punishment and the unconscious and conscious appraisal of emotion are required, as well as systems involved in sustained attention, memory and information-processing. Social interaction requires the ability to abstract rules of behaviour, while the ability to form mental representations of categories of rewarding and aversive stimuli may represent evolutionarily early stages of abstract thought.

These possible links between emotional functions and cognitive processes are of considerable interest in the study of the disease process of schizophrenia, which involves affective blunting, concrete thought processes, impaired function on tests of $\mathrm{cog}$ nitive set-shifting and abnormal categorical thinking.

Damasio, A. R. (1996) The somatic marker hypothesis and the possible functions of the prefrontal cortex. Philosophical Transoctions of the Royal Society of London. Series B: Brological Sciences, 351. 1413-1420.

Taylor, S. F. \& Liberzon, I. (1999) Paying attention to emotion in schizophrenia. British journal of Psychiatry. 174. $6-8$

D. M. Barbenel Imperial College School of Medicine. Department of Psychiatry. Charing Cross Campus, St Dunstan's Road, London W6 8RF

Authors' reply: We are grateful to Barbenel for pointing out an important and complementary approach to the study of emotion and cognition. Whereas we sought in our editorial to emphasise the distinction between cognitive and emotional themes in schizophrenic pathology (Taylor \& Liberzon, 1999), it is equally interesting to understand how emotional responses also have cognitive functions. Barbenel makes the intriguing suggestion that some emotional responses might demonstrate "early stages of abstract thought". As patterned responses, presumably selected by evolutionary pressures, emotions should reflect the output of specific neural systems which adapted to handle recurring situations. As such, specific emotions occur in specific environmental contexts, which clearly delineate categories of behaviour, or rules.

We agree with Barbenel's observation that a disease process affecting cognitive functions, such as set-shifting, and emotional functions, such as affective range, points to both cognition and emotion at the root of pathophysiology. In general, we reject the use of rigid, dichotomous formulations of emotion $v$. cognition, both on theoretical and on empirical grounds. Students of emotion recognise that in spite of their heuristic value as psychological categories, emotion and cognition do not constitute mutually exclusive functional 